\title{
Description of the X(6900) as a Four Charmed Quark State in Terms of a First-Order Tetraquark Mass Formula
}

\author{
Joseph Bevelacqua
}

Funding: The author(s) received no specific funding for this work.

Potential competing interests: The author(s) declared that no potential competing interests exist.

\section{Abstract}

A recently discovered tetraquark candidate having four charmed quarks is investigated using a first-order mass formula. This mass relationship is based on weakly bound meson clusters and provides a reasonable prediction of $6300 \mathrm{MeV} / \mathrm{c} 2$ for the measured $X(6900)$ tetraquark mass.

\subsection{Introduction}

The possibility that hadrons could exist with structures beyond conventional qq or qqq quark configurations was noted by Gell-Mann ${ }^{1}$. Additional theoretical work addressed the basic properties of multi-quark hadrons within an effective quark model of Quantum Chromodynamics (QCD) $)^{2,3}$. More quantitative arguments of multi-quark hadrons utilizing multiquark potentials were studied in lattice QCD ${ }^{4,5}$.

Previous searches of multiquark systems have appeared, but most were later invalidated following additional analysis ${ }^{6}$. However, additional experimental work suggests the existence of both tetraquark ${ }^{7-9}$ and pentaquark $^{10}$ structures. These studies open the possibility of additional exotic structures ${ }^{11}$.

An additional structure has been recently observed in the $\mathrm{J} / \Psi$-pair mass spectrum ${ }^{12}$. A narrow structure at 6.9 Gev/c $c^{2}$ suggests a possible $c c^{\bar{C}} \bar{c}$ structure that could decay into a pair of charmonium states each consisting of a $c^{\bar{C}}$ pair. Ref. 12 proposes the $\mathrm{X}(6900)$ state to be a $c c \bar{c} \bar{c}$ tetraquark. This is the first observation of a fully charmed tetraquark state.

This paper describes the tetraquark candidate X(6900) structure in terms of a first-order mass formula that successfully described the $Z_{C}(3900)^{0}$ and $Z(4430)^{-}$tetraquarks $^{13}$, the $X(5568)$ tetraquark ${ }^{14}$, fusion of heavy mesons into a tetraquark $^{15}$, and proposed $\mathrm{X}(3872)$ tetraquark $^{16}$. The first-order mass formula is a reasonable choice for investing the $\mathrm{X}(6900)$. In addition, the existence of a tetraquark state composed of quark clusters of the same flavor is of theoretical interest.

\subsection{Model Formulation}

Zel'dovich and Sakharov ${ }^{17,18}$ proposed a semiempirical mass formula that provides a prediction of mesons and baryons in terms of effective quark masses. Within this formulation, quark wave functions are assumed to reside in their lowest $1 \mathrm{~S}$ state. These mass formulas are used as the basis for deriving a first-order tetraquark mass formula. In particular, the model utilized in this paper assumes the tetraquark is partitioned into two clusters with the interaction between the clusters providing a minimal contribution to the tetraquark mass. In addition, zero angular momentum is 
assumed to exist between the clusters.

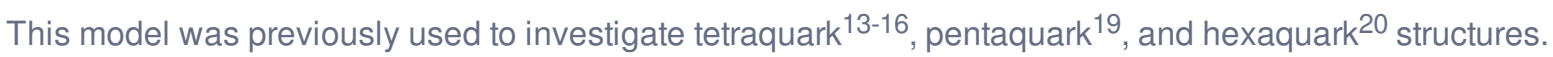

The meson $(\mathrm{m})$ mass $(\mathrm{M})$ formula of Refs. 17 and 18 is:

$M_{m}=\delta_{m}+m_{1}+m_{2}+b_{m}{ }^{\frac{m_{0}^{2}}{m_{1} m_{2}}} \sigma_{1} \cdot \sigma_{2}$

where $m_{1}\left(m_{2}\right)$ are the mass of the first (second) quark comprising the meson, $m_{0}$ is the average mass of a first generation quark $^{21,22}$, and the $\sigma_{i}(i=1$ and 2$)$ are the spin vectors for the quarks incorporated into the meson. The parameters $\delta_{m}$ and $\mathrm{b}_{\mathrm{m}}$ are $40 \mathrm{MeV}$ and $615 \mathrm{MeV}$, respectively ${ }^{18}$.

The last term in Eq. 1 represents the spin-spin interaction of the quarks and $\sigma_{1} \cdot \sigma_{2}$ is the scalar product of the quark spin vectors. $\sigma_{1} \cdot \sigma_{2}$ has the value $-3 / 4,+1 / 4$ for pseudoscalar and vector mesons, respectively ${ }^{18}$.

In formulating the tetraquark mass formula, effective quark masses provided by Griffiths ${ }^{21}$ are utilized. These effective masses for d, u, s, c, b, and t quarks are 340, 336, 486, 1550, 4730, and $177000 \mathrm{MeV} / \mathrm{c}^{2}$, respectively. These masses are utilized in Eq. 1.

These six quarks are arranged in three generations: $[d(-1 / 3), u(+2 / 3)],[s(-1 / 3), c(+2 / 3)]$, and $[b(-1 / 3), t(+2 / 3)]^{22}$. The three generations are specified by the square brackets and the quark charges are given within parentheses.

The quark charges are related to the number of colors $\left(\mathrm{N}_{\mathrm{C}}\right)$ incorporated into the fundamental quantum chromodynamics formulation ${ }^{23,24}$. For example, the first generation quark charges within $\mathrm{SU}\left(\mathrm{N}_{\mathrm{c}}\right)$ are:

$Q_{d}=\frac{1}{2}\left(\frac{1}{N_{c}}-1\right)$

$Q_{u}=\frac{1}{2}\left(\frac{1}{N_{c}}+1\right)$

For conventional QCD using 3 colors, the expected d and u electric charges are obtained. The importance of QCD expansions involving $1 / \mathrm{N}_{\mathrm{C}}$ is outlined in Section 2.1 to illustrate the weak coupling assumption.

The first-order mass formula used in this paper partitions the tetraquark into two meson clusters. Following the suspected X(6900) tetraquark structure, both clusters are assumed to be $c^{\bar{c}}$ mesons (i.e., a $c^{\bar{c}}$ weakly coupled to a $c^{\bar{c}}$ ). Given the simplicity of the first-order mass formula, states having similar configurations (e.g., $c^{\bar{C}}, \bar{C}_{C}, c c$, and $\bar{c} \bar{C}$ ) have the same mass.

Considering the angular momentum assignment for the $\mathrm{J} / \Psi\left(c^{\bar{C}}\right)$ of $\mathrm{J}^{\pi}=1^{-}$, vector plus vector (Eq. 5) meson clusters are used to describe the $\mathrm{X}(6900)$ state. The use of this configuration is the basis for the mass value predicted by the firstorder mass formula.

The weak coupling structure is incorporated to minimize model complexity, which is consistent with an initial firstorder formulation. In general, the tetraquark mass formula is assumed to have the following two forms:

$M_{1}=M_{s m}+M_{s m}+\Phi$

$M_{2}=M_{v m}+M_{v m}+\Phi$

where $\Phi$ defines the interaction between the meson clusters, and $\mathrm{M}_{1(2)}$ represents the scalar (vector) meson clusters defined by Eq. 1. Within the scope of this mass formula, the meson-meson cluster interaction is assumed to be weak and 
sufficiently small to be ignored. Accordingly, Eqs. 4 and 5 represent a quasimolecular four quark system whose basic character is a weakly bound meson-meson system. As noted previously, both clusters are $\mathrm{J} / \Psi, J^{\pi}=1^{-}$vector mesons (i.e., $c_{\bar{C}}$ ). For the $X(6900)$ state, Eq. 5 is used to provide the predicted mass values within the first-order approach.

The mass relationships of Eqs. 1, 4, and 5 do not predict the total angular momentum of the final tetraquark state, but do permit primitive spin coupling to be specified for the individual meson clusters. In addition, the angular momentum between the clusters is assumed to be zero. Specific angular momentum assignments based on the first-order mass formula for the $X(6900)$ state are provided in subsequent discussion.

\subsection{Justification for the Weak Coupling Assumption}

A key assumption of the first-order mass formula of Eqs. 4 and 5 is weak coupling between the two clusters. In particular, the model utilized in this paper assumes the tetraquark is partitioned into two clusters with the interaction between the clusters providing a minimal contribution to the tetraquark mass. Within the scope of this mass formula, the meson-meson cluster interaction is assumed in Eqs. 4 and 5 to be weak and sufficiently small to be ignored.

This assumption is justified because QCD can be investigated as an expansion in $1 / \mathrm{N}_{\mathrm{C}}{ }^{25,26}$. The large $\mathrm{N}_{\mathrm{C}}$ limit reduces to a field theory of weakly interacting meson-like objects. The physical situation with $\mathrm{N}_{\mathrm{C}}=3$ retains many of the characteristics of the $\mathrm{N}_{\mathrm{C}} \rightarrow \infty$ limit, and further justifies the weak coupling approximation.

The $1 / \mathrm{N}_{\mathrm{C}}$ expansion ${ }^{25,26}$ is well accepted in elementary particle physics and leads to the Okubo-Zweig-lizuka (OZI) rule $^{27-29}$ and the Skyrme model ${ }^{30,31}$. In fact, in the $1 / \mathrm{Nc}$ expansion, QCD is reduced to a weakly interacting meson theory, and the meson-meson interaction is regarded to be small25,26. This situation is also a characteristic of Eqs. 4 and 5 .

\subsection{X(6900) Results and Discussion}

The reader should note that the uncertainties in quark masses are likely much larger than any error caused by ignoring the cluster-cluster interaction. Therefore, a detailed quantification or discussion of the cluster-cluster interaction within the first-order mass formula is not warranted.

The $X(6900)$ data did not suggest a spin and parity assignment for this candidate state ${ }^{12}$. Each $\mathrm{J} / \Psi$ meson cluster has a $J^{\pi}=1^{-}$assignment. Given the weak coupling structure with zero angular momentum between the meson clusters, the first-order mass formula has a $\left[\pi^{\pi}(1) \otimes(L=0) \otimes J^{\pi}(2)\right]$ angular momentum structure that reduces to $\left[1^{-} \otimes 1^{-}\right]$or possible $0^{+}, 1^{+}$, and $2^{+}$structures for the $X(6900)$. The notation $J^{\pi}(i)$ for $\mathrm{i}=1$ and 2 represents the two meson clusters comprising the tetraquark.

Eq. 5 is used to calculate the first-order mass formula results corresponding to the possible $X(6900)$ state. The firstorder mass formula predicts $6295 \mathrm{MeV} / \mathrm{c}^{2}$ for the vector meson plus vector meson clusters. Given the accuracy of a firstorder mass formula, this result is necessarily rounded to $6300 \mathrm{MeV} / \mathrm{c}^{2}$. The first-order result is about $9 \%$ lower than the proposed $X(6900)$ value which is consistent with the expected accuracy of a first-order mass formula. This prediction suggests that the $c^{\bar{C}} \bar{C}_{C}$ structure is a possible representation for the proposed X(6900) state.

As noted previously, the predicted X(6900) mass formula underestimates the actual mass, because the model assumes no contribution from the cluster-cluster interaction $(\Phi)$. Since the cluster-cluster interaction creates additional binding, the model will tend to underestimate the tetraquark mass. Given the limited tetraquark data and restrictions of a first-order mass formula, it is not possible to precisely determine the specific value of this energy contribution. Using the 
limited experimental data and first-order mass formula result for the $X(6900)$ suggests that the cluster-cluster interaction contributes about $600 \mathrm{MeV} / \mathrm{c}^{2}\left(6900 \mathrm{MeV} / \mathrm{c}^{2}-6300 \mathrm{MeV} / \mathrm{c}^{2}\right)$ to the charmed tetraquark mass.

\subsection{Conclusions}

The recently discovered tetraquark candidate $\mathrm{X}(6900)$ having the $c^{\bar{c}}{ }_{c} \bar{c}$ structure is investigated using a first-order mass formula incorporating weakly interacting meson clusters. This first-order mass formula predicts that the $X(6900)$ state has a mass of $6300 \mathrm{MeV} / \mathrm{c}^{2}$ which is in reasonable agreement with the measured value. The proposed X(6900) state is also reasonably described by the assumed $c^{\bar{c}} c^{\bar{c}}$ structure.

\section{References}

1) M. Gell-Mann, Phys. Lett. 8, 214 (1964).

2) R. L. Jaffe, Phys. Rev. D15, 267 (1977).

3) R. L. Jaffe, Phys. Rev. D15, 281 (1977).

4) F. Okiharu, H. Suganuma, and T. T. Takahashi, Phys. Rev. D72, 014505 (2005).

5) F. Okiharu, H. Suganuma, and T. T. Takahashi, Phys. Rev. Lett. 94, 192001 (2005).

6) Particle Data Group, J. Phys. G37, 075021 (2010).

7) LHCb Collaboration, Phys. Rev. Lett. 112, 222002 (2014).

8) BESIII Collaboration, Phys. Rev. Lett. 115, 112003 (2015).

9) The D0 Collaboration, arXiv:1602.07588v2 [hep-ex] (2016).

10) LHCb collaboration, Phys. Rev. Lett. 115, 072001 (2015).

11) The ATLAS collaboration, ATLAS-CONF-2015-081 (2015).

12) LHCb Collaboration, Observation of Structure in the J/ $\Psi$-Pair Mass Spectrum, arXiv:2006.16957v1 [hep-ex] 30 June 2020.

13) J. J. Bevelacqua, Physics Essays 29, 198 (2016).

14) J. J. Bevelacqua, Physics Essays 29, 367 (2016).

15) J. J. Bevelacqua, Physics Essays 31, 167 (2018).

16) J. J. Bevelacqua, Physics Essays 32, 469 (2019).

17) Ya. B. Zel'dovich and A. D. Sakharov, Yad. Fiz. 4, 395 (1966).

18) A. D. Sakharov, Sov. Phys. JETP 51, 1059 (1980).

19) J. J. Bevelacqua, Physics Essays 29, 107 (2016).

20) J. J. Bevelacqua, Physics Essays 31, 104 (2018).

21) D. Griffiths, Introduction to Elementary Particles, 2nd ed., (Wiley-VCH, Weinheim, 2008).

22) Particle Data Group, Phys. Rev. D98, 030001 (2018).

23) A. Abbas, Phys. Lett. B 238, 344 (1990).

24) A. Abbas, J. Phys. G 16, L163 (1990).

25) G. 't Hooft, Nucl. Phys. B72, 461 (1974).

26) E. Witten, Nucl. Phys. B160, 57 (1979). 
27) S. Okubo, Phys. Lett. 5, 1975 (1963).

28) G. Zweig, CERN Report No.8419/TH412 (1964).

29) J. lizuka, Prog. Theor. Phys. Suppl. 37, 38 (1966).

30) T.H.R. Skyrme, Proc. R. Soc. A260, 127 (1961).

31) T.H.R. Skyrme, Nucl. Phys. 31, 556 (1962). 